\title{
LINEAR SYNCHROTRON MOTION
}

\author{
S. C. Snowdon \\ June 14,1972
}

\section{PURPOSE}

Since many questions regarding synchrotron phase motion can be answered using a linear approximation for the restoring force, it is of some interest to cast this approximation into the form used in betatron motion. Two examples of the utility of this formalism are given--motion in the adiabatic region and motion near transition.

\section{SYNCHROTRON PHASE MOTION}

In the usual approximation ${ }^{2}$ for synchrotron phase motion the energy gain per turn is

$$
\frac{d E}{d n}=V \sin \left(\int \omega_{R F} d t-h \theta\right)
$$

where $V$ is the peak voltage per turn and $\omega_{R F}$ the angular frequency of the radio frequency, $h$ is the harmonic number and $\theta$ angular position of the particle. Since the revolution frequency is

$$
\frac{\mathrm{dn}}{\mathrm{dt}}=\frac{\omega}{2 \pi}=\frac{\dot{\theta}}{2 \pi}
$$


where $w$ is the angular revolution frequency it is customary to introduce the variable

$$
P=\int \frac{d E}{\omega} \cdot \quad[e V-s e c]
$$

If one also introduces the phase of the particle relative to the radio frequency phase

$$
\phi=h \theta-\int \omega_{R F} d t
$$

one has

$$
\dot{\mathrm{P}}=-\frac{\mathrm{V}}{2 \pi} \sin \phi
$$

and

$$
\dot{\phi}=h \omega-\omega_{R F}
$$

\section{REFERENCE MOTION}

The designation reference motion is used to distinguish between the motion of a particle satisfying Eqs. (5-6) and a particle which is approximately synchronous $\left(h \omega \simeq \omega_{R F}\right)$. Since there are more unknowns in Eqs. (5-6) than equations it is possible to choose the reference motion in many ways. For example, exactly synchronous acceleration will be executed if $\dot{\mathrm{P}}$ and $\mathrm{V}$ are constants. Adiabatic turn-on provides an example in which the reference motion is only approximately synchronous. In any case, if the reference motion is designated by the subscript $R$, one has 


$$
\begin{aligned}
& \dot{P}_{R}=-\frac{V}{2 \pi} \sin \phi_{R} \\
& \dot{\phi}_{R}=h \omega_{R}-\omega_{R F} .
\end{aligned}
$$

The deviation of the general motion from this reference motion may be determined by examining the difference $P-P_{R}$. If, instead of using this difference as a variable, one chooses

$$
P-P_{R}=h J
$$

the variables $(J, \phi)$ will be canonically conjugate. Thus, Eqs. (5-9) give ${ }^{3}$

$$
\begin{aligned}
& \dot{J}=-\frac{V}{2 \pi h}\left(\sin \phi-\sin \phi_{R}\right) \\
& \dot{\phi}=\dot{\phi}_{R}+h\left(\omega-\omega_{R}\right) .
\end{aligned}
$$

Even in this general motion it is customary to linearize Eq. (11) by expanding the actual angular frequency about the reference angular frequency and retain only the first term. Thus one obtains

$$
\begin{aligned}
& \dot{J}=-\frac{V}{2 \pi h}\left(\sin \phi-\sin \phi_{R}\right) \\
& \dot{\phi}=\dot{\phi}_{R}+\frac{h^{2} \omega_{R}^{2}{ }_{R}}{E_{R}} J,
\end{aligned}
$$

where

$$
\kappa_{R}=\frac{\gamma_{T}^{2}-\gamma_{R}^{2}}{\gamma_{T}^{2}\left(\gamma_{R}^{2}-1\right)} .
$$


Here $\gamma_{T}$ is the value of $\gamma_{R}$ at transition and $E_{R}$ is the total energy of the reference particle.

\section{LINEARIZED SYNCHROTRON PHASE MOTION}

If one designates the difference phase $\phi-\phi_{R}$ by

$$
\eta=\phi-\phi_{R}
$$

and expands Eq. (12), retaining only the term linear in $n$ one obtains for the canonically conjugate variables $(J, n)$

$$
\dot{J}=-\frac{V \cos \phi_{R}}{2 \pi h} \eta
$$

and

$$
\dot{n}=\frac{h^{2} \omega_{R}^{2} k_{R}}{E_{R}} \mathrm{~J} .
$$

To bring out the similarity of Eqs. (16-17) with those of betatron motion it is necessary to change the independent variable. Thus, let

$$
s=\int_{0}^{t} \frac{h^{2} \omega_{R}^{2} \kappa_{R}}{E_{R}} d t . \quad\left[(e V-s e c)^{-1}\right]
$$

Then, designating differentiation with respect to $s$ by a prime one has

$$
\begin{aligned}
& J^{\prime}=-\mathrm{Kn} \\
& \eta^{\prime}=\mathrm{J}
\end{aligned}
$$

where 


$$
K=\frac{E_{R} V \cos \phi_{R}}{2 \pi h^{3} \omega_{R}^{2} \kappa_{R}} . \quad\left[(e V-s e c)^{2}\right]
$$

Thus, Eqs. (19-20) become

$$
n^{\prime \prime}+\mathrm{K} n=0
$$

and the similarity to betatron motion is evident. The solution of Eq. (22) may be put in the form of that used in betatron motion. 4

$$
\eta=\sqrt{2 \beta W} \sin \left(\int_{0}^{s} \frac{d s}{\beta}+\gamma\right)
$$

where the constants of the motion $w$, and $\gamma$, have been chosen such that the Jacobian

$$
\frac{\partial(J, \eta)}{\partial(\bar{W}, \gamma)}=1
$$

The function $\beta$ satisfies the second order nonlinear differential equation 4

$$
\frac{1}{2} \beta \beta^{\prime \prime}-\frac{1}{4} \beta^{\prime 2}+\beta^{2} K=1
$$

as is the case in betatron motion. An instantaneous frequency, the synchrotron angular frequency, may be introduced as is clear from the phase in Eq. (23):

$$
\Omega=\frac{1}{\beta} \frac{\mathrm{ds}}{\mathrm{dt}}=\frac{\mathrm{h}^{2} \omega_{\mathrm{R}}^{2} \kappa_{\mathrm{R}}}{\beta \mathrm{E}_{\mathrm{R}}} .
$$




\section{INVARIANT BOUNDARY CURVE}

Since $J=\eta^{\prime}$ one has

$$
J=\sqrt{\frac{2 W}{\beta}}\left[\cos \left(\int_{0}^{s} \frac{d s}{\beta}+\gamma\right)+\frac{1}{2} \beta^{\prime} \sin \left(\int_{0}^{s} \frac{d s}{\beta}+\gamma\right)\right] \cdot[e V-s e c]
$$

Eliminating the phase $\int_{0}^{\mathrm{s}} \frac{\mathrm{ds}}{\bar{\beta}}+\gamma$ between Eq. (23) and (27) one has

$$
W=\frac{1}{2} \beta J^{2}-\frac{1}{2} \beta^{\prime} J \eta+\frac{1}{2 \beta}\left(1+\frac{1}{4} \beta^{\prime 2}\right) n^{2}, \quad[\mathrm{eV}-\mathrm{sec}]
$$

which since $W$ is a constant of the motion, one recognizes Eq. (28) as the invariant curve of courant and snyder. 4 The phase area contained within this boundary ellipse is larea of beam within one bucket not $h$ buckets)

$$
E=\iint \mathrm{dJdn}
$$

But since $(W, Y)$ are canonically related to $(J, \eta), E q \cdot(24)$ gives

$$
E=\iint \mathrm{dWd} \gamma=2 \pi \mathrm{W}
$$

the interpretation being that the phase area associated with the boundary curve is a constant. Thus all the notions related to betatron motion have been carried over to the linearized synchrotron phase motion. One must, however, construct the $\beta$-function for the general case. 


\section{CONSTRUCTION OF THE $\beta-F U N C T I O N$}

Let $\eta_{1}$ and $\eta_{2}$ be two real independent solutions of Eq. (22). Thus

$$
\eta_{1}^{\prime \prime}+\mathrm{kn}_{1}=0 \quad \eta_{2}^{\prime \prime}+\mathrm{k} n_{2}=0 \text {, }
$$

from which one may show that

$$
\frac{\mathrm{d}}{\mathrm{ds}}\left(\eta_{2} \eta_{1}^{\prime}-\eta_{1} \eta_{2}^{\prime}\right)=0 .
$$

Choose, then, any two real independent solutions such that

$$
n_{2} n_{1}^{\prime}-n_{1} n_{2}^{\prime}=1 \text {. }
$$

By direct substitution into Eq. (25) one may show that

$$
\beta=A \eta_{1}^{2}+2 B \eta_{1} \eta_{2}+C \eta_{2}^{2}
$$

is the general solution if

$$
A C-B^{2}=1
$$

where $A, B$, and $C$ are dimensionless constants.

Since the $\beta$-function is related to the boundary curve, the two remaining constants may be found by specifying the initial orientation of the ellipse. 
The properties of the ellipse ${ }^{5}$ are in particular

$$
\begin{aligned}
& \eta_{R}=\sqrt{\frac{\beta E}{\pi}}=\sqrt{2 \beta W} \\
& J_{T}=\frac{1}{2} \frac{\beta^{\prime}}{\beta} \eta_{R}=\frac{1}{2} \beta^{\prime} \sqrt{\frac{2 W}{\beta}} .
\end{aligned}
$$

Hence, if $\eta_{R}(0)$ and $J_{T}(0)$ are given, one may find $\beta(0)$ and $\beta^{\prime}(0)$. Once the specific solutions $\eta_{1}$ and $\eta_{2}$ have been chosen, the constants $\mathrm{A}, \mathrm{B}$, and $\mathrm{C}$ may be found.

\section{SYNCHROTRON MOTION IN ADIABATIC REGION}

The adiabatic regime is defined as motion in which the coefficients in Eqs. (16-17) do not change appreciably in one synchrotron oscillation. This condition is satisfied for synchrotron motion except in the region near transition. Thus under this condition and assuming that one desires to populate the phase plane within the invariant ellipse such that it is approximately upright and does not tumble, the solution of Eq. (25) becomes ( $\beta^{\prime \prime}$ and $\beta^{\prime}$ neglected)

$$
\beta=\frac{1}{\sqrt{K}}=\sqrt{\frac{2 \pi h^{3} \omega_{R}^{2} K_{R}}{E_{R} \operatorname{Vos} \phi_{R}}} .
$$

Eq. (23) becomes

$$
\eta=\sqrt{\frac{2 W}{\sqrt{k}}} \sin \left(\int_{0}^{t} \Omega d t+\gamma\right)
$$

where Eq. (26) gives 


$$
\Omega=\omega_{R} \sqrt{\frac{h k_{R} V \cos \phi_{R}}{2 \pi E_{R}}} .
$$

Eq. (39) demonstrates that the adiabatic damping of the phase oscillation varies as

$$
\sqrt{\beta}=\sqrt[4]{\mathrm{K}}
$$

\section{SYNCHROTRON MOTION INCLUDING TRANSITION}

Herrera ${ }^{6}$ has calculated this motion recently. It is of interest to see how it fits into the preceding formalism. His basic assumption apart from using a linear theory is that the reasonable variation of $\gamma$ near transition (here taken to be at $t=0$ )

$$
\gamma=\gamma_{T}+\dot{\gamma}_{T} t
$$

giving

$$
\frac{\mathrm{h}^{2} \omega_{\mathrm{R}}^{2} \mathrm{~K}}{\mathrm{E}_{\mathrm{R}}}=-\mathrm{Mt}
$$

where

$$
M=\frac{2 h^{2} \omega_{T}^{2} \dot{\gamma}_{T}}{E_{T} \gamma_{T}\left(\gamma_{T}^{2}-1\right)} \quad\left[(e V)^{-1}-\sec ^{-3}\right]
$$

provides an $M$ which may be taken as constant for all times. Thus, Eq. (18) becomes

$$
s=-\frac{1}{2} M t^{2}
$$


Eq. (2l) becomes ( $V$ and $\phi_{R}$ assumed constant)

$$
K(s)=\frac{V \cos \phi_{R}}{2 \pi h \sqrt{M}} \cdot \frac{1}{\sqrt{-s}} \quad\left[(e V-s e c)^{2}\right]
$$

If the reference phase changes from $\phi_{\mathrm{R}}$ prior to transition to $\pi-\phi_{R}$ just after transition, Eq. (46) is valid both above and below transition if $\phi_{R}$ is the reference phase below transition at the "time" (-s) away from transition. For convenience let

$$
a=\sqrt{\frac{\sqrt{\cos \phi_{R}}}{2 \pi h \sqrt{M}}} \quad\left[(e V-s e c)^{3 / 4}\right]
$$

Eq. (22) becomes

$$
\eta^{\prime \prime}+\frac{\mathrm{a}^{2}}{\sqrt{-\mathrm{s}}} \eta=0
$$

Two independent solutions which satisfy Eq. (33) are

$$
\eta_{1}=\sqrt{\frac{2 \pi}{3}}(-s)^{1 / 2} \mathrm{~J}_{2 / 3}(\mathrm{v})
$$

and

$$
\eta_{2}=\sqrt{\frac{2 \pi}{3}}(-s)^{1 / 2} \mathrm{~N}_{2 / 3}(\mathrm{v})
$$

where

$$
v=\frac{4 a}{3}(-s)^{3 / 4}
$$

Eq. (34) gives

$$
\beta=\frac{2 \pi}{3}(-s)\left[\mathrm{AJ}_{2 / 3}^{2}(\mathrm{v})+2 \mathrm{BJ}_{2 / 3}(\mathrm{v}) \mathrm{N}_{2 / 3}(\mathrm{v})+\mathrm{CN}_{2 / 3}^{2}(\mathrm{v})\right]
$$


The choice of $\mathrm{A}, \mathrm{B}$, and $\mathrm{C}$ is made as follows. One wants the boundary curve to be approximately an upright ellipse for large $(-\mathrm{s})$. For large $(-\mathrm{s}) \mathrm{Eq}$. (52) becomes

$$
\beta=\frac{1}{2 a}(-s)^{1 / 4}\left[A+C+2 B \sin \left(2 v-\frac{7 \pi}{6}\right)+(A-C) \cos \left(2 v-\frac{7 \pi}{6}\right)\right]
$$

For no tumbling the coefficients of the trigonometric terms must be zero. Thus $B=0$ and $A=C$. Eq. (35) then gives
$A=1$
$B=0$
$\mathrm{C}=1$.

Thus

$$
\beta=\eta_{1}^{2}+\eta_{2}^{2}=\frac{2 \pi}{3}(-s)\left[J_{2 / 3}^{2}(v)+N_{2 / 3}^{2}(v)\right]
$$

Near transition, Eq. (55) becomes

$$
\beta=\frac{8 \pi}{9}\left(\frac{3}{2 a}\right)^{4 / 3} \frac{1}{\Gamma^{2}\left(\frac{1}{3}\right)}+\frac{2}{\sqrt{3}}(-s)+\ldots
$$

Hence, at transition

$$
\begin{aligned}
& \beta(0)=\frac{8 \pi}{9}\left(\frac{3}{2 a}\right)^{4 / 3} \frac{1}{\Gamma^{2}\left(\frac{1}{3}\right)} \\
& \beta^{\prime}(0)=-\frac{2}{\sqrt{3}}
\end{aligned}
$$

For the NAL booster one has approximately

$$
\begin{aligned}
\mathrm{V} & =654 \mathrm{kV} / \text { turn } \\
\phi_{\mathrm{R}} & =70^{\circ} \\
\gamma_{\mathrm{T}} & =5.4458 \\
\mathrm{E}_{\mathrm{T}} & =5.1096 \mathrm{GeV}
\end{aligned}
$$




$$
\begin{aligned}
\mathrm{h} & =84 \\
\dot{\gamma}_{\mathrm{T}} & =396.6 / \mathrm{sec} .
\end{aligned}
$$

For these parameters

$$
\begin{aligned}
\beta(0) & =7.08 / \mathrm{eV} / \mathrm{sec} \\
\beta^{\prime}(0) & =-1.15
\end{aligned}
$$

Detailed numerical studies by Lee $^{7}$ following the trapping and subsequent acceleration of many particles indicates that the trapped particles indeed may be put into an upright ellipse and that at transition the ellipse is characterized by

$$
\begin{aligned}
\beta(0) & =7.26 / \mathrm{eV} / \mathrm{sec} \\
\beta^{\prime}(0) & =-1.19
\end{aligned}
$$

in reasonable agreement with the analytical results above. 


\section{REFERENCES}

1. L.C. Teng, Unified Formulation for Transverse and Longitudinal Oscillations in a Synchrotron, National Accelerator Laboratory, TM-320, August 24, 1971.

2. K.R. Symon and A.M. Sessler, Methods of Radio Frequency Acceleration with Applications to High Current and Intersecting Beam Accelerators, MURA-106, April 16, 1956.

3. S.C. Snowdon, Booster Constant Bucket Area Regime with Space Charge, National Accelerator Laboratory, TM-304, May 24, 1971.

4. E.D. Courant and H.S. Snyder, Theory of the Alternating Gradient Synchrotron, Annals of Physics $\underline{3}, 1$ (1958).

5. C. Bovet, R. Gouiran, I. Gumowski, and K.H. Reich, A Selection of Formulae and Useful Data for the Design of A.G. Synchrotrons, CERN/MPS-SI/Int. DL/70/4, April 23, 1970.

6. J.C. Herrera, Theory of Synchrotron Motion near Transition in an AGS, Particle Accelerators 3, 49 (1972).

7. W.W. Lee, Private Communication; for background see: W.W. Lee and L.C. Teng, Beam Bunch Length Matching at Transition Crossing with Space Charge Forces, IEEE Trans. on Nuclear Science NS-18, 1057 (1971). 